\title{
INFLUÊNCIA DO AUXÍLIO MECÂNICO NA COLHEITA DE TOMATES ${ }^{1}$
}

\section{AUGUSTO C. SANCHEZ ${ }^{2}$, MARCOS D. FERREIRA ${ }^{3}$, ANA M. DE MAGALHÃES ${ }^{4}$, OSCAR A. BRAUNBECK ${ }^{5}$, LUIS A. B. CORTEZ ${ }^{6}$, PAULO S. G. MAGALHÃES ${ }^{7}$}

\begin{abstract}
RESUMO: O objetivo deste trabalho foi avaliar parâmetros que influenciam na colheita auxiliada e comparar a colheita tradicionalmente realizada para tomate de mesa em propriedades agrícolas, no Estado de São Paulo. Para tanto, foi desenvolvido um dispositivo de auxílio à colheita, de estrutura metálica, dotado de rodas e com assentos acoplados para um ou dois colhedores, avaliado em quatro modalidades de colheita: testemunha (colheita-padrão utilizada na Fazenda Santa Luzia, Brotas-SP), colhedor a pé com auxílio, colhedor sentado com auxílio e composição dos dois sistemas, com dois colhedores sentados e um a pé, em diferentes velocidades de deslocamento. Os resultados demonstraram que os tipos de colheita com auxílio mecânico se apresentaram mais eficientes do que a testemunha em relação à produtividade horária média por colhedor. O sistema de colheita auxiliada com três colhedores apresentou aumento de $290 \%$ na produtividade média horária por colhedor, para a faixa de 0,5 a 1,0 fruto por planta, seguido dos sistemas de colheita auxiliada com colhedor a pé, com aumento de aproximadamente $41 \%$, e colheita auxiliada com colhedor sentado, com aumento de aproximadamente $35 \%$. Esses resultados comprovam a importância do uso do dispositivo de auxílio mecânico.
\end{abstract}

PALAVRAS-CHAVE: Lycopersicum esculentum, colhedor, produtividade.

\section{INFLUENCE OF THE HARVESTING AID EQUIPMENT IN TOMATO HARVEST}

\begin{abstract}
Fresh tomato harvest is traditionally made without harvesting aids. The main goal of this research was to evaluate performance parameters of fresh tomato harvesting aid equipment and compare it to traditional harvest, in the state of São Paulo. Therefore, an equipment was developed and the harvest process was evaluated in four different ways: traditional system (harvest system used in Santa Luzia farm, Brotas, SP, Brazil), picker walking with a harvesting aid equipment, picker seated in a harvesting aid equipment and a composition of both systems: two pickers seated and one picker walking in two different velocities ranges. The different systems using harvesting aid showed an average yield by picker more efficient than reference. Harvest system using three pickers showed an increase of $290 \%$ on yield average by picker, on the range of $0.5-1.0$ fruit per plant, followed by the systems with a walking picker, that increased productivity in $41 \%$, and picker seated harvester, that showed an increase of 35\%. These results demonstrate the importance of using a harvesting aid equipment.
\end{abstract}

KEYWORDS: Lycopersicum esculentum, harvester, productivity.

\section{INTRODUÇÃO}

O tomateiro é a segunda hortaliça cultivada no mundo e, em quantidade produzida, é superada apenas pela batata (FAO, 2006; CANÇADO JÚNIOR et al., 2003). A produção mundial de tomate em 2005 foi de aproximadamente 125 milhões de toneladas, com o Brasil ficando em nono lugar, com produção de 3,3 milhões de toneladas (FAO, 2006), sendo o Estado de São Paulo o segundo maior produtor, com aproximadamente 700 mil toneladas, das quais $60 \%$ foi destinada para o consumo in natura (IEA, 2006; AGRIANUAL, 2006; TSUNECHIRO et al., 2005).

\footnotetext{
${ }^{1}$ Parte do Projeto Unidade Móvel de Auxílio à Colheita - UNIMAC (financiado pela FAPESP).

${ }^{2}$ Técnico Mecânico, Estudante de graduação, FEAGRI/UNICAMP, Campinas - SP, augusto.sanchez@agr.unicamp.br.

${ }^{3}$ Prof. Doutor, Área de Concentração Tecnologia Pós-Colheita, FEAGRI/ UNICAMP, Campinas - SP.

${ }^{4}$ Eng ${ }^{\mathrm{a}}$ Agrícola, Mestranda, FEAGRI/UNICAMP, Campinas - SP.

${ }^{5}$ Prof. Doutor, Área de Concentração Máquinas Agrícolas, FEAGRI/UNICAMP, Campinas - SP.

${ }^{6}$ Prof. Doutor, Área de Concentração Construções Rurais e Ambiência, FEAGRI/UNICAMP, Campinas - SP.

${ }^{7}$ Prof. Doutor, Área de Concentração Máquinas Agrícolas, FEAGRI/UNICAMP, Campinas - SP.

Recebido pelo Conselho Editorial em: 10-6-2005

Aprovado pelo Conselho Editorial em: 19-4-2006
} 
O tomate para consumo in natura é cultivado com tutoramento, ou seja, estaqueado ou envarado, caracterizado por colheitas múltiplas, enquanto o tomate para o consumo industrial é cultivado sem tutoramento, em cultivo rasteiro, com colheita única. Em conseqüência dessa especificidade, as cultivares de tomate são melhoradas, visando ao local, à forma de cultivo e à finalidade para consumo (CANÇADO JÚNIOR et al., 2003).

No caso do tomate de mesa, a colheita é realizada manualmente sem equipamentos de auxílio (SANCHEZ et al., 2003). Alguns estudos foram realizados utilizando equipamentos adaptados da colheita de tomate industrial, porém os resultados obtidos não tiveram muito sucesso, principalmente devido à realização de colheita única e não múltipla, com conseqüente redução na produtividade (25-47\% menor se comparada à colheita manual) (STOFELLA \& SHERMAN, 1984). HOOD et al. (1975) relatam que o uso de colhedoras é viável para o tomate de mesa, pois proporcionam maior rendimento e diminuição das perdas pós-colheita; todavia, a colheita manual é mais vantajosa, pois possibilita colheitas múltiplas.

O manuseio excessivo na colheita tradicional influencia na perda da qualidade dos frutos, com aumento significativo na percentagem de danos físicos e perda de massa. Essa perda na qualidade foi confirmada por meio da avaliação final do produto, após armazenamento de 21 dias, em que 22,5\% dos frutos apresentavam danos físicos e podridão (FERREIRA et al., 2004).

O objetivo deste trabalho foi avaliar o uso de um dispositivo de auxílio à colheita, propondo a realização de colheitas múltiplas e em condições adaptadas às atualmente utilizadas em campo, variando o número de colhedores, visando a reduzir o tempo requerido para a colheita, bem como o manuseio e a incidência de danos físicos.

\section{MATERIAL E MÉTODOS}

Os ensaios foram realizados na Fazenda Santa Luzia, localizada no município de Brotas - SP. A escolha desse local foi motivada principalmente pela distância entre linhas $(1,60 \mathrm{~m})$, favorável ao trânsito de um pequeno veículo na plantação, pelo satisfatório nivelamento das ruas (inclinações máximas de $5 \%)$, pelo espaçamento uniforme entre plantas $(0,50 \mathrm{~m})$ e ainda por serem essas conduzidas em fios verticais. $\mathrm{O}$ equipamento de auxílio à colheita utilizado consiste de uma estrutura metálica dotada de rodas, com assentos acoplados para um ou dois colhedores, sendo conduzido por um microtrator, marca GOLDONI Base 20, com tração nas quatro rodas e motor diesel com potência de $16 \mathrm{~kW}(22 \mathrm{cv})$ a $3.000 \mathrm{rpm}$ (Figuras 1 e 2).

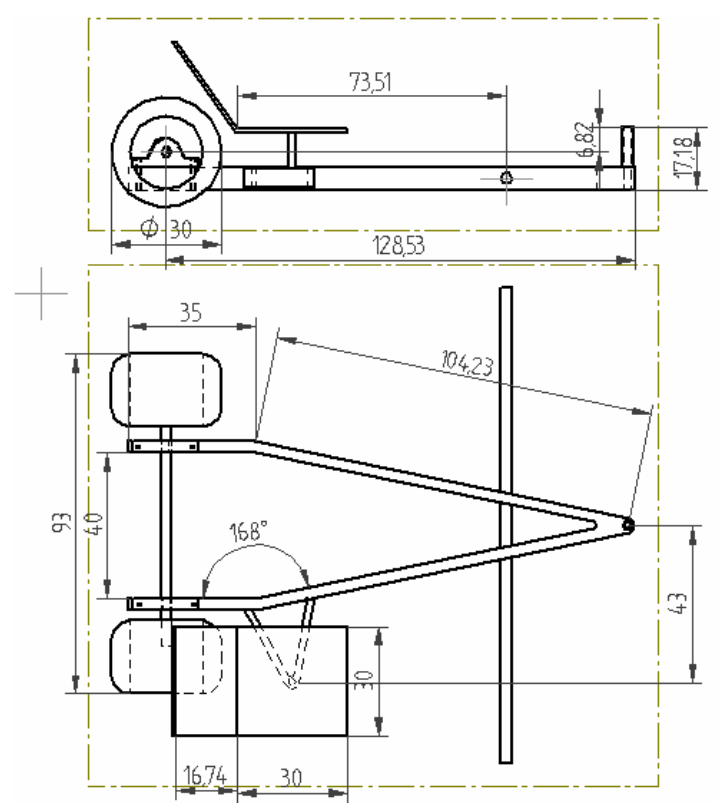

FIGURA 1. Croqui da vista lateral e superior do dispositivo de auxílio à colheita com suas respectivas dimensões $(\mathrm{cm})$. 


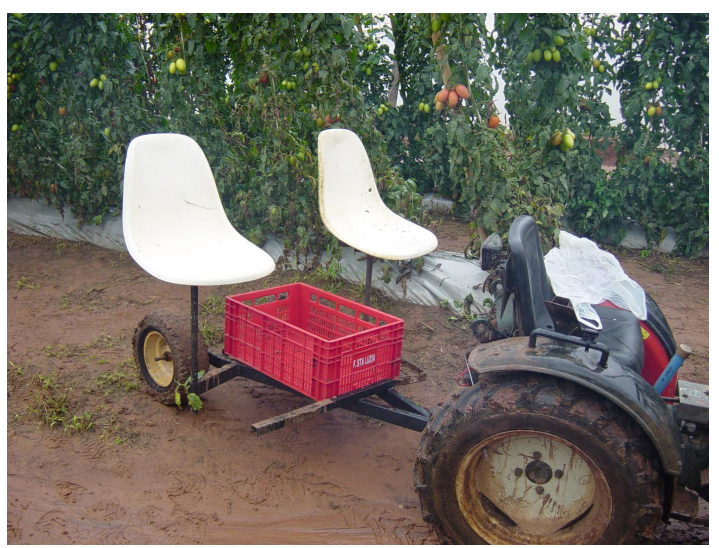

FIGURA 2. Dispositivo de auxílio à colheita com assentos acoplados para dois colhedores.

Foram avaliados quatro sistemas de colheita: (1) Testemunha: sistema de colheita realizado na Fazenda Santa Luzia. O colhedor de tomates caminha entre as ruas colhendo frutos maduros que são depositados em caixas plásticas, com dimensões externas de $0,55 \mathrm{~m}$ de comprimento, 0,30 m de altura e 0,35 m de largura, que se encontram em uma gôndola com dimensões de 1,04 x 0,58 x $0,75 \mathrm{~m}$; conforme a colheita é realizada, o colhedor vai empurrando essa gôndola nas ruas; (2) Auxiliado a pé: o colhedor acompanha o dispositivo de auxílio móvel, colhendo os frutos e colocando-os sobre esse; (3) Auxiliado sentado: o colhedor fica sentado sobre o dispositivo de auxílio; (4) Auxiliado com três colhedores: consiste na configuração mista dos dois ensaios anteriores, ou seja, dois colhedores operando sentados e um terceiro caminhando atrás do dispositivo de auxílio, colhendo os frutos que não foram colhidos pelos outros dois. Os testes foram realizados por colhedores da própria Fazenda Santa Luzia, experientes em cultivo de tomate destinado para mesa, cultivar Débora Plus (Sakata Seeds Co.), em distâncias de 50 m (50 plantas), em três repetições. As ruas foram escolhidas aleatoriamente em cada sistema testado, a fim de que não houvesse influência da quantidade média de frutos por planta nos resultados.

Os parâmetros avaliados foram a velocidade média do trajeto, o aproveitamento percentual e a produtividade horária por colhedor.

A velocidade média do trajeto foi determinada por meio do tempo gasto pelo(s) colhedor(es) para percorrer a distância de $50 \mathrm{~m}$.

Pela relação entre o número de frutos colhidos e o número de frutos deixados de colher na primeira passagem, foi possível determinar o aproveitamento percentual de cada ensaio, de acordo com a eq.(1).

$$
\mathrm{AP}=\frac{\mathrm{Fc}}{(\mathrm{Fc}+\mathrm{Fnc})} 100
$$

em que,

AP - aproveitamento percentual, \%;

$\mathrm{Fc}$ - número de frutos colhidos, $\mathrm{e}$

Fnc - número de frutos deixados de colher.

Por meio da massa de cada caixa plástica contendo os frutos colhidos, do tempo gasto na colheita e do número de colhedores em cada linha, foi possível estimar a produtividade horária de cada ensaio e a produtividade horária de cada colhedor, de acordo com as eqs.(2) e (3).

$$
\begin{aligned}
& \mathrm{Ph}=\frac{\mathrm{Mfc}}{\mathrm{t}^{\prime}} \\
& \mathrm{Pc}=\frac{\mathrm{Ph}}{\mathrm{Nc}}
\end{aligned}
$$

em que, 
$\mathrm{Ph}$ - produtividade horária de cada ensaio, $\mathrm{kg} \mathrm{h}^{-1}$;

Mfc - massa de cada caixa com os frutos colhidos, $\mathrm{kg}$;

t' - tempo gasto na colheita, $\mathrm{h}$;

$\mathrm{Pc}$ - produtividade horária de cada colhedor, $\mathrm{kg} \mathrm{h}^{-1}$, e

$\mathrm{Nc}$ - número de colhedores.

O número de frutos por plantas foi determinado por meio da eq.(4).

$$
\mathrm{Fp}=\left(\frac{\mathrm{Fc}+\mathrm{Fnc}}{\mathrm{NPt}-\mathrm{Pf}}\right)
$$

em que,

Fp - frutos por planta;

$\mathrm{Fc}$ - frutos colhidos;

Fnc - frutos deixados de colher;

$\mathrm{Npt}$ - número de plantas teoricamente existentes, e

Pf - plantas faltantes, mortas ou doentes.

Os resultados obtidos para a velocidade média do trajeto, aproveitamento percentual e produtividade horária por colhedor foram diferenciados em duas faixas de colheita de 0,5 a 1,0 fruto por planta para todos os tratamentos e de 1,0 a 1,5 fruto por planta para os tratamentos com dispositivo de auxílio.

Para a análise estatística dos dados coletados, foi utilizado o programa computacional STATGRAPHICS Plus 4.1. O delineamento utilizado foi o inteiramente casualizado, em esquema fatorial, e para a comparação das médias, utilizou-se do teste de Tukey, a 5\% de probabilidade.

\section{RESULTADOS E DISCUSSÃO}

As maiores médias para a velocidade do trajeto $\left(28,64\right.$ e $\left.29,53 \mathrm{~m} \mathrm{~min}^{-1}\right)$ foram obtidas para o tratamento de colheita auxiliada com três colhedores para as duas faixas de colheita de 0,5 a 1,0 e de 1,0 a $1,5(\mathrm{Fp})$, respectivamente, apresentando diferença estatística a $5 \%$ de probabilidade em relação aos demais tratamentos (Tabelas 1 e 2). Na faixa de 1,0 a 1,5 (Fp), a testemunha apresentou velocidades inferiores à estipulada $\left(5 \mathrm{~m} \mathrm{~min}^{-1}\right)$, e por isso não foi considerada para essa faixa.

O sistema auxiliado sentado apresentou-se como o mais eficiente na faixa de 0,5 a 1,0 (Fp), com aproveitamento percentual de 98,05\% (Tabela 1). Na faixa de 1,0 a 1,5 (Fp), a maior média $(88,46 \%)$ foi obtida pelo sistema auxiliado a pé; todavia, esse sistema não se diferenciou estatisticamente do sistema auxiliado sentado, ou seja, nessa faixa, os dois sistemas foram satisfatórios (Tabela 2).

O sistema de colheita auxiliado com três colhedores não mostrou bom aproveitamento percentual, aproximadamente $75 \%$ nas duas faixas de colheita (Tabelas 1 e 2), o que pode ser explicado pela grande quantidade de frutos que foram deixados de colher na primeira passagem, ocasionados pelas altas velocidades apresentadas pelo sistema de auxílio.

TABELA 1. Análise do teste de Tukey para a velocidade média do trajeto e para o aproveitamento percentual, na faixa de 0,5 a 1,0 fruto por planta.

\begin{tabular}{lcl}
\hline \multirow{2}{*}{ Tratamentos } & \multicolumn{2}{c}{ Médias* } \\
\cline { 2 - 3 } & $\mathrm{V}\left(\mathrm{m} \mathrm{min}^{-1}\right)$ & AP $(\%)$ \\
\hline Testemunha & $8,53 \mathrm{a}$ & $94,03 \mathrm{bc}$ \\
Auxiliado sentado & $10,48 \mathrm{a}$ & $98,05 \mathrm{c}$ \\
Auxiliado a pé & $10,86 \mathrm{a}$ & $92,87 \mathrm{~b}$ \\
Auxiliado com três colhedores & $28,64 \mathrm{~b}$ & $75,62 \mathrm{a}$ \\
\hline
\end{tabular}

* médias seguidas de mesma letra nas colunas não diferem entre si, a 5\% de probabilidade. 
TABELA 2. Análise do Teste de Tukey para a velocidade média do trajeto e para o aproveitamento percentual na faixa 1,0 a 1,5 fruto por planta.

\begin{tabular}{lcc}
\hline \multirow{2}{*}{ Tratamentos } & \multicolumn{2}{c}{ Médias* } \\
\cline { 2 - 3 } & $\mathrm{V}\left(\mathrm{m} \mathrm{min}^{-1}\right)$ & $\mathrm{AP}(\%)$ \\
\hline Auxiliado a pé & $11,72 \mathrm{a}$ & $88,46 \mathrm{~b}$ \\
Auxiliado sentado & $12,96 \mathrm{a}$ & $84,19 \mathrm{~b}$ \\
Auxiliado com três colhedores & $29,53 \mathrm{~b}$ & $73,11 \mathrm{a}$ \\
\hline
\end{tabular}

* médias seguidas de mesma letra nas colunas não diferem entre si, a $5 \%$ de probabilidade.

A produtividade horária por colhedor mostrou diferença significativa a $5 \%$ de probabilidade para as duas faixas de 0,5 a 1,0 e 1,0 a 1,5 fruto por planta. $O$ sistema de colheita auxiliado com três colhedores apresentou os melhores resultados, com valor médio de $240 \mathrm{~kg} \mathrm{~h}^{-1}$ para a faixa de 0,5 a 1,0 (Fp), equivalendo ao aumento de $290 \%$, quando comparado à testemunha (Figura 3 ).

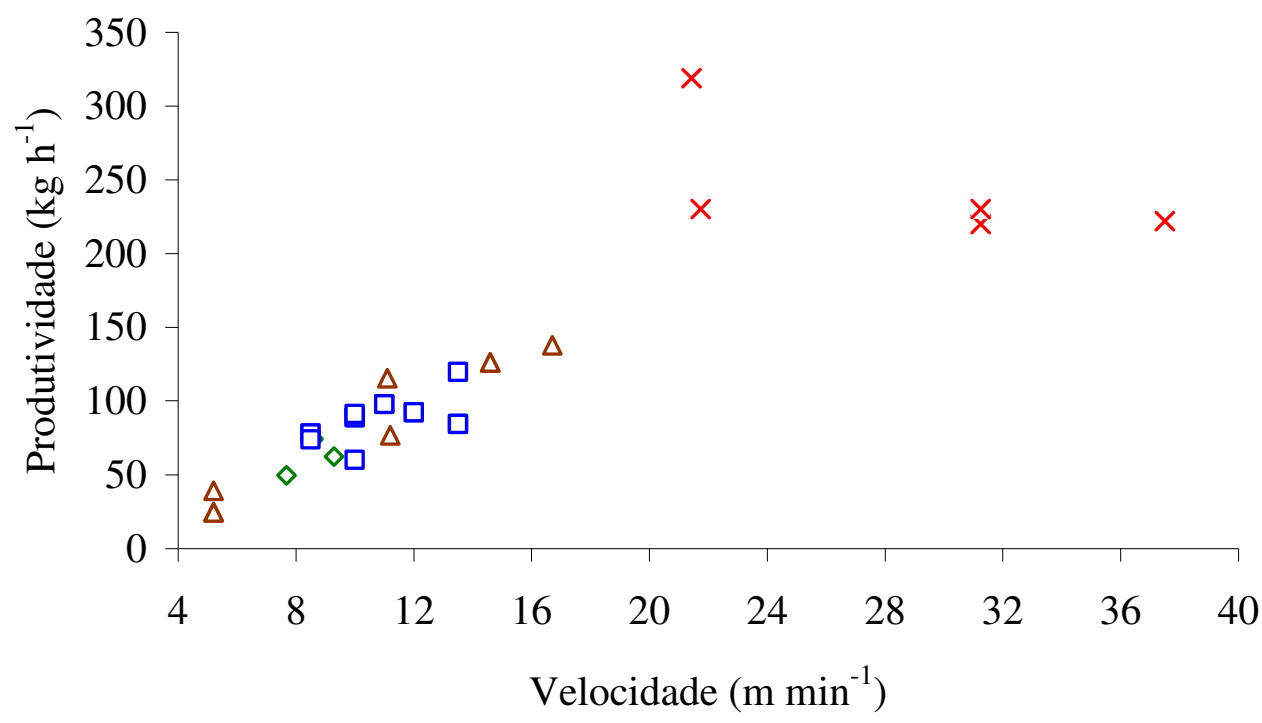

$\triangle$ Testemunha $\square$ Auxiliado a pé $\Delta$ Auxiliado sentado $\times$ Auxiliado 3 colhedores

FIGURA 3. Produtividade horária por colhedor entre os sistemas de colheita de tomate de mesa, para a faixa de 0,5 a 1,0 fruto por planta.

Para a faixa de 1,0 a 1,5 (Fp), o sistema auxiliado com três colhedores apresentou aumento superior a $60 \%$ em relação aos outros dois sistemas auxiliados (Figura 4). Esses resultados foram obtidos devido à repassagem realizada pelo terceiro colhedor a pé, justificando a importância do repasse nessa condição.

Nos demais sistemas, as maiores médias, nas duas faixas de frutos por planta, foram obtidas pelo sistema de colheita auxiliada com o colhedor a pé. Na faixa de 0,5 a 1,0 (Fp), esse sistema apresentou aumento de $41 \%$ na produtividade horária por colhedor, quando comparado à testemunha, $3 \%$ em relação ao sistema de colheita com o colhedor sentado para essa mesma faixa e 4,4\% para a faixa de 1,0 a 1,5 fruto por planta (Figuras 3 e 4). Esses resultados ocorreram devido à maior mobilidade do colhedor. 


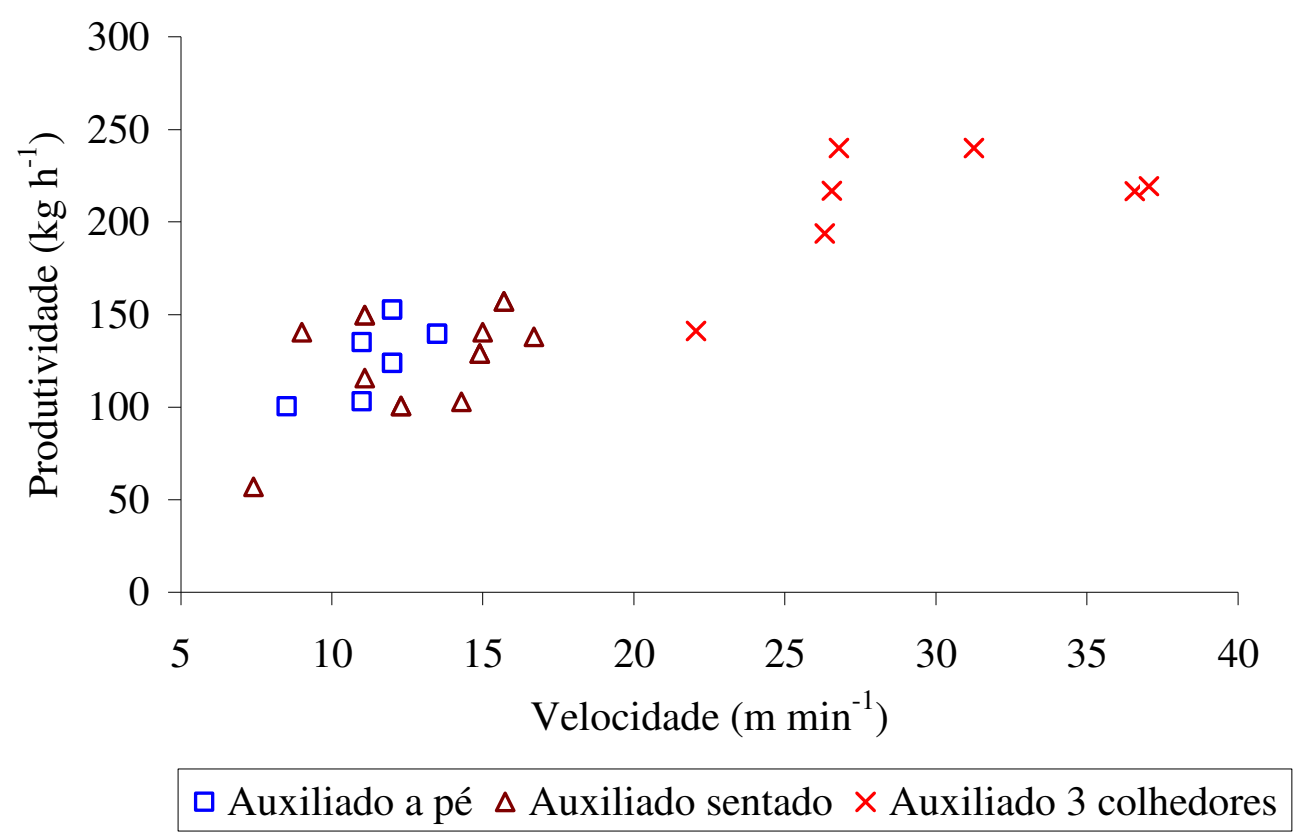

FIGURA 4. Produtividade horária por colhedor entre os sistemas de colheita de tomate de mesa, para faixa de 1,0 a 1,5 fruto por planta.

\section{CONCLUSÕES}

Entre os métodos de colheita avaliados, os sistemas auxiliados mostraram-se mais eficientes, apresentando maior aproveitamento percentual e maior produtividade horária. O método de colheita auxiliada com três colhedores pode ser considerado o melhor, pois apresentou resultados superiores de produtividade horária. Por meio de melhor adequação de trabalho do dispositivo e treinamento dos operadores, pode-se aumentar a eficiência da colheita com maior aproveitamento percentual.

Para futuros trabalhos, sugere-se um comparativo de custos entre o sistema convencional e o auxiliado, avaliando-se a viabilidade econômica de implantar um sistema de colheita auxiliada.

\section{AGRADECIMENTOS}

Os autores agradecem aos funcionários da Fazenda Santa Luzia, aos funcionários da Faculdade de Engenharia Agrícola da UNICAMP e à FAPESP, que financia o projeto "Proposta de um Novo Sistema (UNIMAC), visando à Melhoria da Qualidade e Diminuição das Perdas PósColheita em Tomate de Mesa" (UNIMAC, Unidade Móvel de Auxílio à Colheita).

\section{REFERÊNCIAS}

AGRIANUAL 2006: anuário estatístico da agricultura brasileira. São Paulo: Instituto FNP Consultoria \& Comércio, 2006. 504 p.

CANÇADO JÚNIOR, F.L.; CAMARGO FILHO, W.P.; ESTANISLAU, M.L.L.; PAIVA, B.M.; MAZZEI, A.R.; ALVES, H.S. Tomate para mesa. Informe Agropecuário, Belo Horizonte, v.24, n.219, p.7-18, 2003.

FERREIRA, M.D.; FRANCO, A.T.O.; NOGUEIRA, M.F.M.; ALMEIDA, R.V.C.; TAVARES, M. Avaliação da etapa da colheita em tomates de mesa cv. Débora. Brazilian Journal of Food Technology, Campinas, v.7, n.2, p.173-8, 2004.

FOOD AND AGRICULTURE ORGANIZATION OF THE UNITED NATIONS - FAO, FAOSTAT. Database. Disponível em: <http://faostat.fao.org>. Acesso em: 27 fev. 2006. 
HOOD, C.E.; WEBB, B.K.; ALPER, Y.; SIMS JR., E.T.; JOBES, R.A. A new fresh market tomato harvesting system. Transactions of the ASAE, St. Joseph, v.18, n.6, p.1021-6, 1975.

INSTITUTO DE ECONOMIA AGRÍCOLA - IEA. Banco de dados. Disponível em: $<$ http://www.iea.sp.gov.br>. Acesso em: 27 fev. 2006.

SANCHEZ, A.C.; FERREIRA, M.D.; BRAUNBECK, O.A.; BRYAN, N. Sistema Unimac Comparação de produtividade entre duas cultivares de tomate de mesa na colheita com auxílio mecânico. In: WORKSHOP TOMATE NA UNICAMP, 1., 2003, Campinas. Anais ... Campinas: Faculdade de Engenharia Agrícola, UNICAMP, 2003. 1 CD-ROM.

STOFELLA, P. J.; SHERMAN, M. Semi-mechanically harvested fresh-market tomato yields as influenced by harvest date and cultivar. HortScience, Alexandria, v.19, n.5, p.679-81, 1984.

TSUNECHIRO, A.; COELHO, P.J.; CASER, D.V.; AMARAL, A.M.P.; MARTINS, V.A.; BUENO, C.R.F.; GHOBRIL, C.N. Valor da produção agropecuária do Estado de São Paulo em 2005: estimativa preliminar. Informações Econômicas, São Paulo, v.35, n.10, p.42-52, 2005. 